\title{
Aharonov-Bohm interference as a probe of Majorana fermions
}

\author{
T. C. Bartolo $\odot,{ }^{1, *}$ J. S. Smith $\odot,{ }^{1}$ B. Muralidharan, ${ }^{2}$ C. Müller $\odot,{ }^{3,4}$ T. M. Stace, ${ }^{4}$ and J. H. Cole $\odot^{1, \dagger}$ \\ ${ }^{1}$ Chemical and Quantum Physics, School of Science, RMIT University, Melbourne, Victoria 3000, Australia \\ ${ }^{2}$ Department of Electrical Engineering, Indian Institute of Technology Bombay, Powai, Mumbai 400076, India \\ ${ }^{3}$ IBM Quantum, IBM Research - Zurich, 8803 Rüschlikon, Switzerland \\ ${ }^{4}$ ARC Centre for Engineered Quantum System, School of Mathematics and Physics, University of Queensland, \\ Brisbane, Queensland 4072, Australia
}

(Received 29 June 2020; revised 1 October 2020; accepted 28 November 2020; published 28 December 2020)

\begin{abstract}
Majorana fermions act as their own antiparticle, and they have long been thought to be confined to the realm of pure theory. However, interest in them has recently resurfaced, as it was realized through the work of Kitaev that some experimentally accessible condensed matter systems can host these exotic excitations as bound states on the boundaries of one-dimensional chains, and that their topological and non-Abelian nature holds promise for quantum computation. Unambiguously detecting the experimental signatures of Majorana bound states has turned out to be challenging, as many other phenomena lead to similar experimental behavior. Here, we computationally study a ring comprised of two Kitaev model chains with tunnel coupling between them, where an applied magnetic field allows for Aharonov-Bohm interference in transport through the resulting ring structure. We use a nonequilibrium Green's function technique to analyze the transport properties of the ring in both the presence and absence of Majorana zero modes. Further, we show that these results are robust against weak disorder in the presence of an applied magnetic field. This computational model suggests another signature for the presence of these topologically protected bound states can be found in the magnetic field dependence of devices with loop geometries.
\end{abstract}

DOI: 10.1103/PhysRevResearch.2.043430

\section{INTRODUCTION}

Majorana fermions were postulated in 1937 as fermionic excitations that act as their own antiparticle [1], but so far they have not been experimentally shown to exist in nature. More recently it was shown theoretically that one-dimensional (1D) systems exhibiting $p$-wave superconductivity within certain parameters host Majorana zero modes (MZMs) at their boundaries [2]. It was soon realized that the topological nature of these bound states make them ideal candidates for quantum computation, as it helps to protect them from some types of decoherence [3,4]. Destroying information encoded in this way requires a global perturbation that is strong enough to break the topologically nontrivial phase of the system [5,6]. Further, their non-Abelian character [2,7] allows one to manipulate pairs of MZMs through braided exchange of their relative positions, showing a way towards topologically protected quantum computation [2,3,8-10]. One possible experimental system hosting MZMs is one-dimensional (1D) semiconductor wires with strong spin-orbit coupling and proximity induced superconductivity $[2,11]$. Several recent

\footnotetext{
*tommy.bartolo@gmail.com

†jared.cole@rmit.edu.au
}

Published by the American Physical Society under the terms of the Creative Commons Attribution 4.0 International license. Further distribution of this work must maintain attribution to the author(s) and the published article's title, journal citation, and DOI. works have reported experimental evidence for the existence of MZMs in such condensed matter systems [11-17]. However there exist a number of confounding effects that have signatures similar to MZMs [17-23] and which make the unambiguous detection of Majorana fermions an ongoing challenge.

The standard theory model that allows for MZMs is the Kitaev model nanowire [2]. It consists of a 1D tight binding chain with proximity induced $p$-wave superconductivity, as illustrated in Fig. 1(a). Several different materials have been considered to realize such wires [2,11-17]. Although the system parameters vary between the different materials, for all these systems the key physics can be approximated by the effective Kitaev nanowire Hamiltonian.

The parameter regimes of the Kitaev nanowire have been extensively studied [2,24-26], as have its transport properties [27-29]. By coupling two Kitaev nanowires through nonsuperconducting links at their ends, we form an AharonovBohm (AB) ring, as illustrated in Fig. 1(b).

Different ring geometries have previously been investigated using interferometry, from which it was suggested that the periodicity of the conductance as a function of magnetic field might be used as a way to identify Majorana bound states [30-33]. Other studies employed either scattering matrix theory or Green's function techniques to study a normal $\mathrm{AB}$ ring containing a single nanowire hosting MZMs [34-38]. For example, the transport properties of such an $\mathrm{AB}$ ring were found to be sensitive to the difference between MZMs and Andreev bound states [34,39-44]. Studies of two-dimensional systems of finite extent have investigated interference effects 
(a)

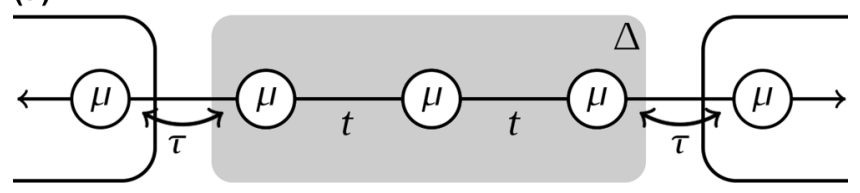

(b)

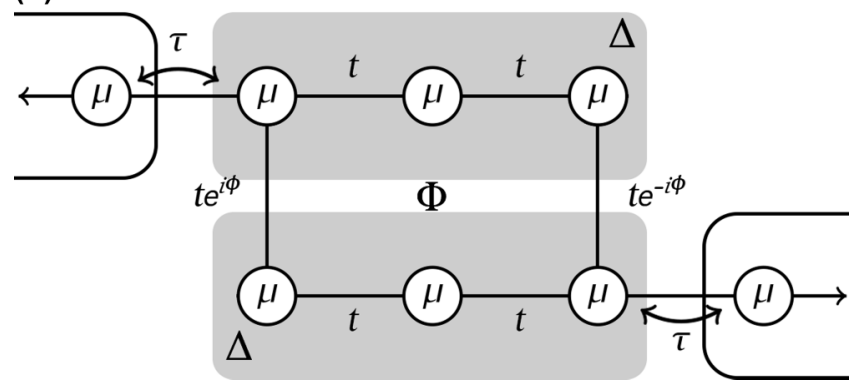

FIG. 1. A diagram of a 1D Kitaev nanowire (a) and AharonovBohm ring (b). These consist of a tight binding chain with onsite potential $\mu$, hopping strength $t$, and a $p$-wave pairing amplitude of $\Delta$. Normal leads are coupled to the nanowire with a hopping amplitude of $\tau$.

due to chiral Majorana fermion edge states at the normalsuperconductor boundary $[13,45,46]$.

In this paper we study the interplay between the $\mathrm{AB}$ effect and MZMs, and expand on these previous works by analyzing the transport characteristics of an $\mathrm{AB}$ ring formed by two coupled Kitaev nanowires. We employ the nonequilibrium Green's function (NEGF) formalism and explore the relationship between model parameters and transport characteristics for a finite size ring geometry. Previous studies have investigated the case of MZMs in a finite nanowire $[47,48]$. Such models are particularly useful as all experimentally realizable devices are finite, which limits what can be understood simply from the bulk properties of the materials hosting MZMs. The relation between $\mathrm{AB}$ interference and Majorana bound states in such a loop geometry has previously been studied using a scattering matrix approach with the wide-band approximation [49]. However, energy resolved transport characteristics of such a circuit provide important clues on how such effects can be probed experimentally and will help us to further understand the experimental signatures of these topologically nontrivial bound states. Here we show that mapping the energy resolved transmission through an $\mathrm{AB}$ ring comprising two MZMs displays the expected resonance at zero energy. However, mapping this resonance as a function of magnetic field, on-site potential, or superconducting order parameter results in characteristic responses, suggesting the possibility of unambiguously distinguishing MZMs from trivial bound states.

The paper proceeds as follows: Section II introduces the standard toy model for the Kitaev nanowire. The NEGF formalism is then outlined in Sec. II A. Section III expands on the Kitaev nanowire by introducing an $\mathrm{AB}$ ring comprised of two coupled Kitaev nanowires. Finally, Sec. IV examines the transport properties of this ring in the presence of an applied magnetic field. We conclude in Sec. VI.
TABLE I. The different parameter regimes for the Kitaev nanowire. These regimes are obtained from the Hamiltonian shown in Eq. (1).

\begin{tabular}{lc}
\hline \hline Regime & Model parameters \\
\hline Topologically trivial & $\mu / t>2$ \\
Topologically nontrivial & $\mu / t<2, \Delta / t \neq 0$ \\
Superconducting & $\Delta / t \gg 1$ \\
Normal & $\Delta / t=0$ \\
\hline \hline
\end{tabular}

\section{THE KITAEV NANOWIRE}

We begin by following Kitaev in defining a model Hamiltonian $\left(\hat{H}_{\mathrm{KC}}\right)$ for a system admitting the existence of MZMs as

$$
\begin{aligned}
\hat{H}_{\mathrm{NW}} & =\sum_{j=1}^{\mathrm{N}}\left[-t\left(c_{j}^{\dagger} c_{j+1}\right)-\mu\left(c_{j}^{\dagger} c_{j}-\frac{1}{2}\right)+\text { H.c. }\right], \\
\hat{H}_{\mathrm{KC}} & =\hat{H}_{\mathrm{NW}}+\sum_{j=1}^{\mathrm{N}}\left[\Delta e^{i \theta} c_{j} c_{j+1}+\Delta e^{-i \theta} c_{j+1}^{\dagger} c_{j}^{\dagger}\right]
\end{aligned}
$$

where $t$ is the nearest-neighbor hopping strength, $\mu$ is the on-site potential, $\Delta$ is the $p$-wave pairing amplitude, $\theta$ is the superconducting phase and $N$ is the number of sites in the chain. Without loss of generality we set $\theta=2 \pi n$, where $n \in$ $\mathbb{Z}$, resulting in $e^{i \theta}=1 . c_{j}$ is the fermionic annihilation operator acting on the $j$ th site. We write a particle-hole symmetric (PHS) form of the Hamiltonian through the BogoliubovdeGennes (BdG) Hamiltonian:

$$
H_{\mathrm{BdG}}=\left[\begin{array}{cc}
H_{\mathrm{NW}} & \Delta \\
-\Delta^{*} & -H_{\mathrm{NW}}^{*}
\end{array}\right] \text {. }
$$

$H_{\mathrm{NW}}$ is the Hamiltonian for a normal wire and $\Delta$ is the $p$-wave pairing amplitude between particles and holes. For more details see Appendix A. Following Chen et al. [24] we model the Kitaev nanowire as a 1D tight binding chain that consists of 50 sites with the on-site potential $\mu$ and $p$-wave pairing amplitude $\Delta$ as adjustable parameters. The essential physics studied here remains unchanged with the length of the chain, so long as the chain is long enough such that the ground-state wave functions do not overlap, which is the requirement for topologically nontrivial MZMs. To calculate the electrical response of the 1D Kitaev nanowire, we couple it to normal leads on either side and apply a nonequilibrium Green's function (NEGF) technique, as described in Sec. II A.

The Kitaev Hamiltonian has three different regimes which are of interest to us here. These are the topologically trivial, the topologically nontrivial, and the superconducting regimes. The different model parameters for each of these regimes are summarized in Table I, where we also list parameters for the normal (nonsuperconducting) regime. Figure 2 shows how the eigenspectrum of this Hamiltonian depends on the value of $\mu / t$. In this figure we see the separation of the topologically trivial and nontrivial regimes at approximately $\mu / t=2$. When $\mu / t<2$ the system is topologically nontrivial and we have a degenerate ground state at $E / t=0$. In the topologically trivial regime, when $\mu / t>2$, the ground state 


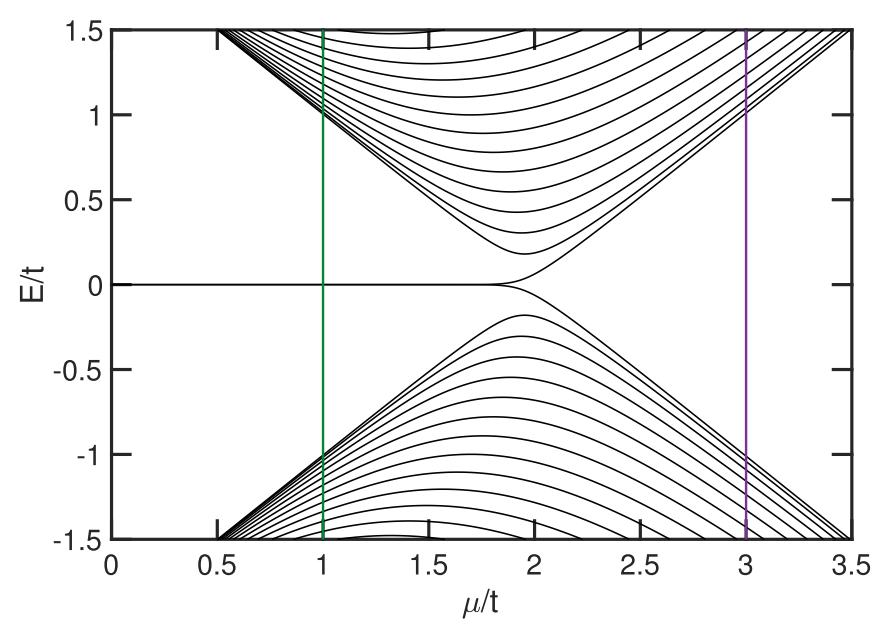

FIG. 2. Eigenenergies for a 1D Kitaev nanowire as a function of $\mu / t$ with $\Delta / t=1.0$. Vertical lines indicate values of $\mu / t$ used to compute the transmission probabilities in Fig. 3.

of the system is no longer degenerate, nor does it appear at $E / t=0$.

\section{A. Nonequilibrium Green's function formalism}

To calculate the electrical response of a Kitaev nanowire we apply the NEGF formalism [50], which allows us to compute transmission probabilities as a function of energy. We are also thereby able to introduce open boundary conditions and model transport through the device with changing magnetic field. The retarded Green's function for the device $G^{\mathrm{R}}$, from which the transport properties of the system can be calculated, is written as

$$
G^{\mathrm{R}}(E)=\left[\left(E+i 0^{+}\right) \mathbb{I}-H_{\mathrm{BdG}}-\Sigma_{\mathrm{L}}(E)-\Sigma_{\mathrm{R}}(E)\right]^{-1}
$$

where $E$ is energy and $0^{+}$is a small positive number. For brevity we suppress the energy dependence in subsequent Green's functions, transmission functions, and other operators. The Hamiltonian describing the device is modified by two self-energy terms $\Sigma_{L}$ and $\Sigma_{R}$ to include the left and right leads respectively. These self-energy terms are calculated from the hopping parameter and onsite potential for the leads and are used to model the open boundaries. The nearestneighbor hopping strength between the leads and the device is given by $\tau / t=0.3$. These weak links $(\tau<t)$ at the interfaces, together with the self-energy terms, modify the system's density of states such that resonant tunneling dominates transport through the device. The Green's function is used along with the Caroli formula [Eq. (4)] to calculate transmission probabilities for particles and holes moving through the device. When applying the NEGF formalism to a conventional charge transport problem, the transmission probability $T$ is given by

$$
T=\operatorname{Tr}\left(\Gamma_{\mathrm{L}} G^{\mathrm{R}} \Gamma_{\mathrm{R}} G^{\mathrm{A}}\right)
$$

with $G^{\mathrm{A}}=\left(G^{\mathrm{R}}\right)^{\dagger}$ and broadening matrices $\Gamma_{\alpha}$ given by

$$
\Gamma_{\alpha}=i\left(\Sigma_{\alpha}-\Sigma_{\alpha}^{\dagger}\right)
$$

with $\alpha$ an index describing the left (L) and right (R) contacts.
The conductance $g$ can be calculated via [51]

$$
g=\frac{-2 q^{2}}{h} \int_{-\infty}^{\infty} T(E) \frac{\partial f(E)}{\partial E} d E,
$$

where $h$ is Planck's constant, $q$ is the charge of the particle, and $f(E)$ is the equilibrium Fermi-Dirac distribution function. In the limit as temperature goes to zero, the conductance is simply proportional to the transmission function and therefore in everything that follows we only consider $T(E)$. In general if a voltage bias is applied to the device then the onsite potential becomes spatially dependent and the integral in Eq. (6) must be taken over a range of energies. Although these complications are important in discussing real devices, they do not change the underlying physics and so we focus on the zero-bias limit. For the BdG Hamiltonian we must now account for both electron and hole degrees of freedom. We can compute the total transmission probability as the sum of the transmission probabilities for direct transmission $T_{\mathrm{D}}$, Andreev reflection $T_{\mathrm{A}}$, and crossed Andreev reflection $T_{\mathrm{CA}}$ :

$$
T^{\mathrm{e}(\mathrm{h})}=T_{\mathrm{D}}^{\mathrm{e}(\mathrm{h})}+T_{\mathrm{A}}^{\mathrm{e}(\mathrm{h})}+T_{\mathrm{CA}}^{\mathrm{e}(\mathrm{h})}
$$

with

$$
\begin{aligned}
& T_{\mathrm{D}}^{\mathrm{e}(\mathrm{h})}=\operatorname{Tr}\left(\Gamma_{\mathrm{L}}^{\mathrm{e}(\mathrm{h})} G^{\mathrm{R}} \Gamma_{\mathrm{R}}^{\mathrm{e}(\mathrm{h})} G^{\mathrm{A}}\right), \\
& T_{\mathrm{A}}^{\mathrm{e}(\mathrm{h})}=\operatorname{Tr}\left(\Gamma_{\mathrm{L}}^{\mathrm{e}(\mathrm{h})} G^{\mathrm{R}} \Gamma_{\mathrm{L}}^{\mathrm{h}(\mathrm{e})} G^{\mathrm{A}}\right), \\
& T_{\mathrm{CA}}^{\mathrm{e}(\mathrm{h})}=\operatorname{Tr}\left(\Gamma_{\mathrm{L}}^{\mathrm{e}(\mathrm{h})} G^{\mathrm{R}} \Gamma_{\mathrm{R}}^{\mathrm{h}(\mathrm{e})} G^{\mathrm{A}}\right) .
\end{aligned}
$$

Equation (7) therefore gives the transmission probability of a particle (hole) through the device at a particular energy (see Appendix B for further details).

The broadening matrices are now represented as a $2 \times 2$ matrix due to PHS:

$$
\Gamma_{\alpha}=\left[\begin{array}{cc}
\Gamma_{\alpha}^{(\mathrm{e})} & \Gamma_{\alpha}^{(\mathrm{eh})} \\
\Gamma_{\alpha}^{(\mathrm{he})} & \Gamma_{\alpha}^{(\mathrm{h})}
\end{array}\right],
$$

where the superscript represents the type of particles involved in the interaction. For example (e) is a electron-electron interaction and $(\mathrm{h})$ is a hole-hole interaction. As the system has normal leads we do not consider the off-diagonal terms which correspond to electron-hole interaction in the broadening matrices, i.e., $\Gamma_{\alpha}^{(\mathrm{he})}=\Gamma_{\alpha}^{(\mathrm{eh})}=0$.

As we are ultimately interested in the total conductance through the device, in what follows we often plot the combined transmission due to electrons and holes, $\left(T^{\mathrm{e}}+T^{\mathrm{h}}\right) / 2$, where the individual electron and hole transmission functions are given by Eq. (7).

\section{B. Signatures of Majorana fermions and the zero-bias anomaly}

A signature of MZMs in the electrical response of these systems is the existence of a zero-bias anomaly (ZBA) that is topologically protected [14]. This ZBA is a conduction mode at $E / t=0$ that results from the zero-energy ground state of the MZMs. Figure 3 shows the transmission probability for the 1D Kitaev nanowire in the topologically trivial and nontrivial regimes. The figure shows the transmission probability for a particle through the Kitaev nanowire at $E / t=0$, with on-site potentials $\mu / t=1$ ( $\mu / t<2$, topologically nontrivial) and $\mu / t=3(\mu / t>2$, topologically trivial $)$. The presence of 


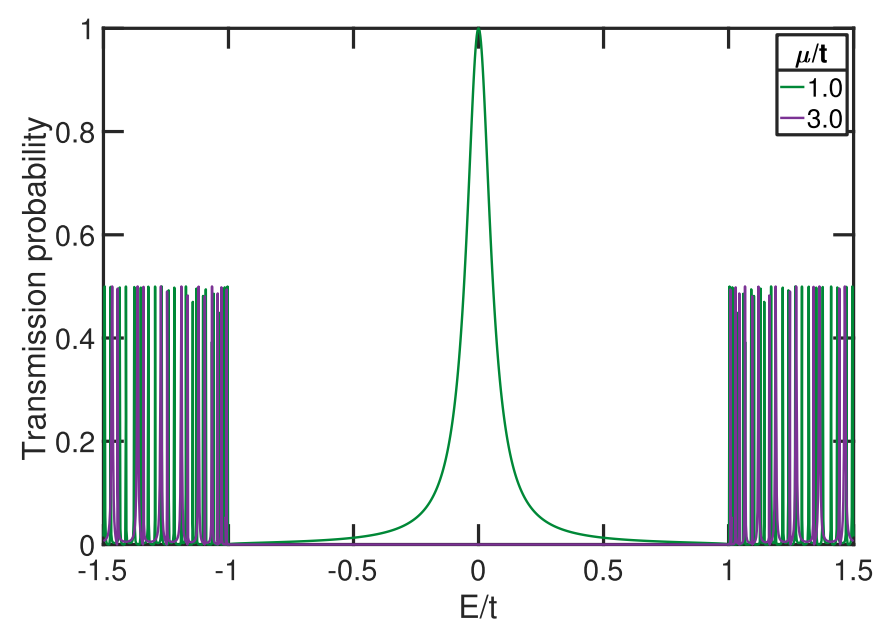

FIG. 3. Transmission probability as a function of energy for the 1D Kitaev nanowire for two values of $\mu / t$, highlighted in Fig. 2 as vertical lines. The nanowire has a $p$-wave pairing amplitude of $\Delta / t=1.0$ and a hopping strength between the leads and the device of $\tau / t=0.3$.

a nonzero probability for transmission of a particle at $E / t=0$ when $\mu / t=1$ is characteristic of the ZBA [11]. Here the width of the zero-energy peak in the transmission probability is directly related to the strength of the coupling to the device, $\tau$. As $\tau$ increases there is a stronger connection between the device and the leads, which creates a broader resonance peak [50]. In the topologically trivial regime $(\mu / t=3)$ there is no zero-energy peak as all states of the Hamiltonian fall outside of the energy gap, as seen in Fig. 3.

\section{THE AHARONOV-BOHM RING}

We now consider a device comprised of two 1D Kitaev nanowires, as illustrated in Fig. 1. This system is capable of supporting two pairs of MZMs (one MZM at each of the four interfaces between the device and the leads). For all AB ring devices modelled here we use a ring comprised of two Kitaev nanowires with 50 sites,

$$
H_{\mathrm{AB}}=\left[\begin{array}{cc}
H_{\mathrm{BdG}} & t e^{-i \phi} \Pi \\
t e^{i \phi} \Pi & H_{\mathrm{BdG}}
\end{array}\right]
$$

where the off-diagonal blocks $\Pi$ are the adjacency matrices defined such that they couple the BdG Hamiltonians at the edges of the wires, as per Fig. 1(b), and $\phi$ is the Peierls phase for an applied magnetic field.

The topological phase diagram for this $\mathrm{AB}$ ring is equivalent to the topological phase diagram of the 1D Kitaev nanowire. The presence of a ZBA for the AB ring can been seen in Fig. 4 for each of the parameter regimes described in Table I. The two Kitaev nanowires of the ring are connected by a nearest-neighbor hopping strength of $t^{\prime}$, as shown in Fig. 1(b). In all that follows we set $t^{\prime}=t_{1}=t_{2}=t$. The $p$ wave pairing amplitude between the two nanowires is zero. This corresponds to the case of normal tunneling between two superconducting nanowires.

We now apply our NEGF method to an $\mathrm{AB}$ ring. Figure 5 shows the transmission probability for an $\mathrm{AB}$ ring at energies close to $E=0 t$ and values of the on-site potential

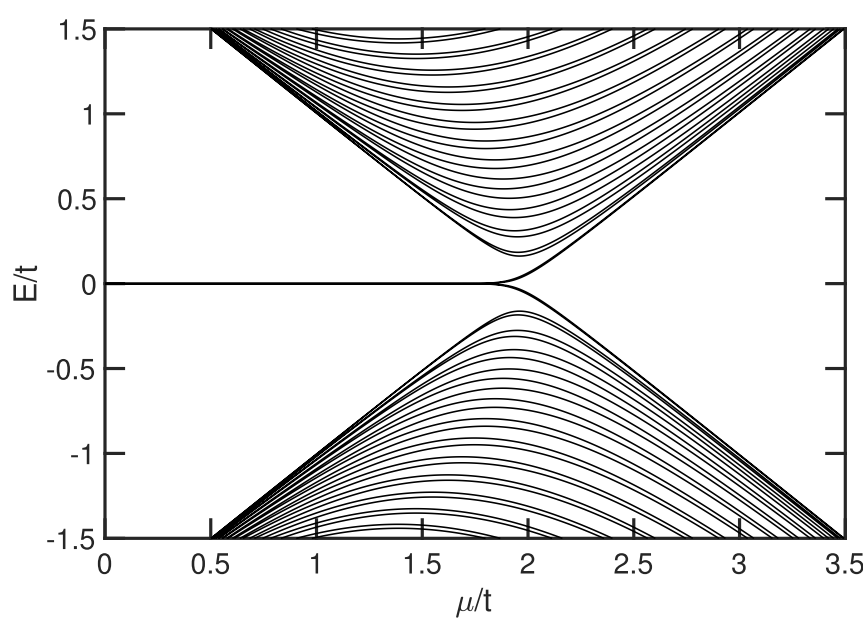

FIG. 4. Eigenenergies for an $\mathrm{AB}$ ring (without leads attached) as a function of $\mu / t$ with $\Delta / t=1.0$. Here the magnetic flux through the ring is $\Phi / \Phi_{0}=0$.

$\mu / t=\{1.775,1.925,1.950,2.000\}$. For $\mu / t>1.775$ we see peaks form due to the topologically trivial quasiparticle states which lie outside the superconducting energy gap. As $\mu$ is decreased, we see the formation of a peak in the transmission probability at zero energy. The appearance of the ZBA correlates with the system becoming topologically nontrivial. At $\mu / t=1.775$ the zero-energy transmission is maximized, which suggests that at this value of the on-site potential the system is in the topologically nontrivial regime. The value of $\mu$ at which the system becomes topologically nontrivial is affected by the number of sites. As the number of sites increases the system approaches the ideal case and the value tends to $\mu / t \approx 2$. For relatively small numbers of sites, the value of $\mu / t$ corresponding to a topological phase transition is reduced due to finite size effects.

By examining each of the individual components of Eq. (7) [given in Eqs. (8)-(10)] we are able to see how each

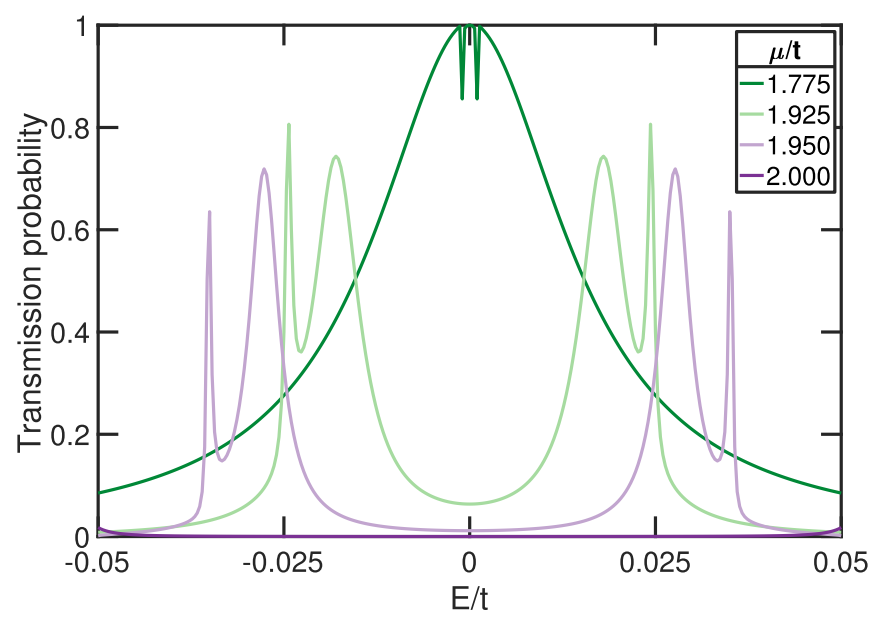

FIG. 5. Transmission probability of an $\mathrm{AB}$ ring at energies about zero for several different values of the on-site potential $\mu$. The $\mathrm{AB}$ ring has a $p$-wave pairing amplitude $\Delta / t=1.0$ and normal leads are attached with a coupling strength of $\tau / t=0.3$. The external applied magnetic field strength here is $\Phi / \Phi_{0}=0$. 

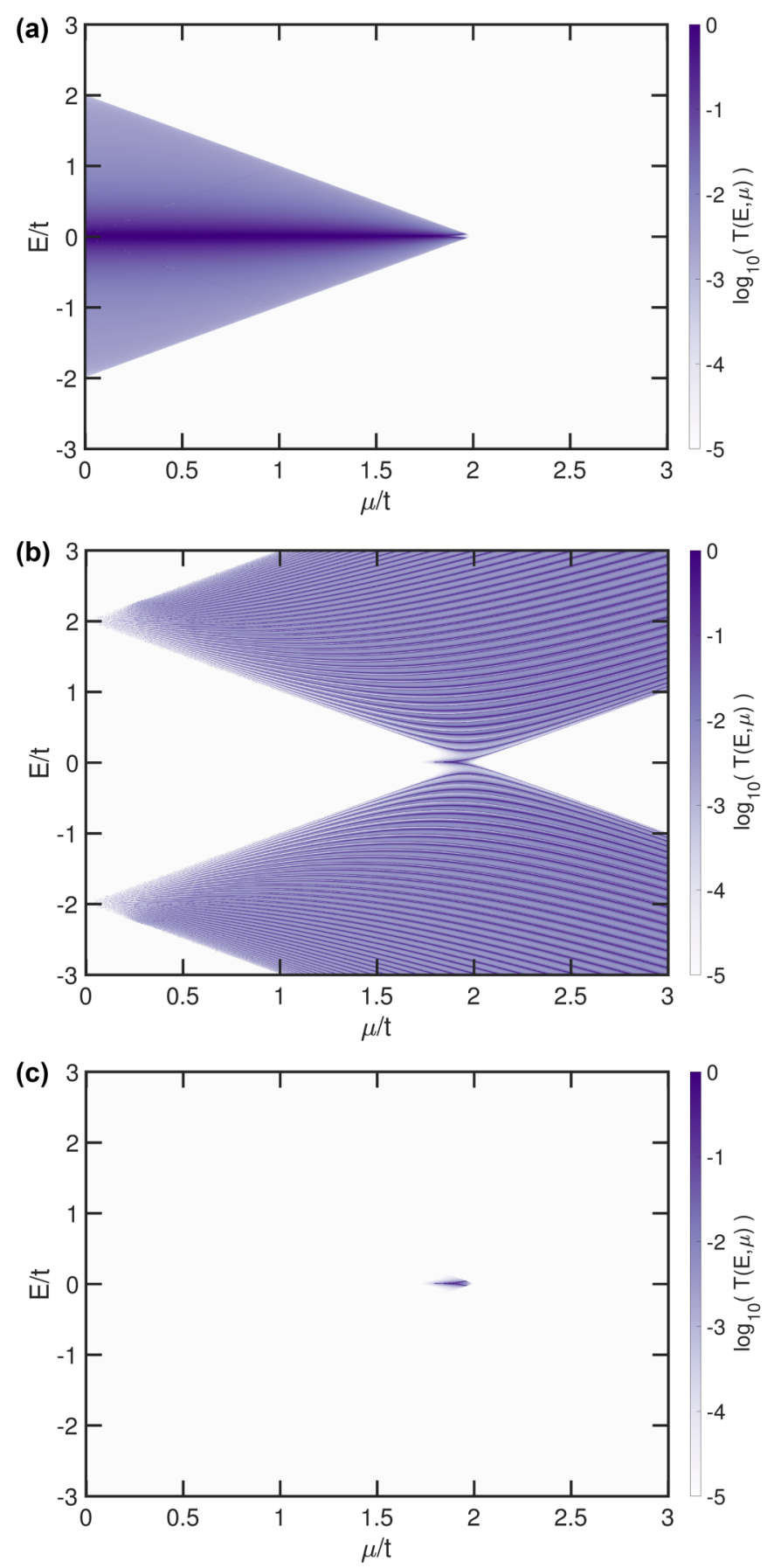

FIG. 6. Contributions to the transmission probability from (a) Andreev, (b) direct, and (c) crossed Andreev transmission for an $\mathrm{AB}$ ring with $50 \times 2$ sites as a function of energy $E$ and on-site potential $\mu$. Here the magnetic flux through the ring is $\Phi / \Phi_{0}=0$. The $\mathrm{AB}$ ring has a $p$-wave pairing amplitude $\Delta / t=1.0$ and normal leads are attached with a coupling strength of $\tau / t=0.3$. Note the logarithmic color scale used in order to resolve the weaker transmission probabilities in (b) and (c).

component of the transmission probability influences the total transmission probability through the device. It also allows for insight into how the magnetic field induced interference affects each of the difference types of transmission (direct, Andreev, and crossed Andreev). Figure 6 shows the trans- mission probability as a function of energy $E$ and on-site potential $\mu$ for Andreev transmission, direct transmission, and crossed Andreev transmission with a magnetic flux through the ring of $\Phi / \Phi_{0}=0$. We see in Fig. 6(a) that the subgap states are due entirely to resonant Andreev transmission, while in Fig. 6(b) we see that direct transmission occurs via states above and below the gap. Figure 6(c) shows that the crossed Andreev transmission only exists close to the point of transition between trivial and nontrivial topological behavior, at zero energy and $\mu / t \approx 2$. The crossed Andreev transmission is relatively weak compared to the other contributions.

\section{MAGNETIC FIELD APPLIED TO AN AHARONOV-BOHM RING}

We now apply a perpendicular magnetic field to the $A B$ ring by Peierls substitution to observe interference in the transmission probability [52-54]. We choose a gauge such that the Peierls phase $\Phi$ (equivalent to magnetic flux through the ring and given here in units of flux quanta $\Phi_{0}=h / 2 e$ ) drops across only the normal links. By applying a magnetic field to the $\mathrm{AB}$ ring in the topologically trivial and nontrivial regimes, we see the changes in the transmission probability as a function of magnetic flux due to the presence (or absence) of the MZMs.

Resonances in the transport spectrum of the ring are caused by weak links between the device and the leads [27]. Applying a magnetic field to the $\mathrm{AB}$ ring in the normal regime $(\mu / t=1, \Delta / t=0)$, as in Fig. 7(a), we see that there is completely destructive interference at $\Phi / \Phi_{0}= \pm \frac{\pi}{2}$, which results in the transmission probability going to zero at these values of magnetic flux. The symmetry of the transmission resonances in Fig. 7 about zero energy follows directly from the PHS. One important consideration is whether such a system would destroy superconductivity in the ring by applying a magnetic field larger than the critical field. To investigate this we can consider a ring of niobium titanium nitride as an example. If the ring has an area of $1 \mu \mathrm{m}^{2}$, one magnetic flux quanta corresponds to $2 \mathrm{mT}$, which is considerably smaller than a typical critical field of approximately $28 \mathrm{mT}$. This would allow the mapping of $\mathrm{AB}$ interference for many oscillation periods without collapsing the superconducting state $[55,56]$.

In the case where $\Delta / t=0.05$ and $\mu / t=1$, as in Fig. 7(b), we see the formation of a sub-gap state that oscillates in energy, as a function of magnetic flux, with a period of $\pi$. Note that the value of $\Delta / t$ is not large enough to overcome finite size effects here and that the transport spectrum is characterized by a lack of the zero-energy state at $\Phi / \Phi_{0}=0$. Furthermore for a magnetic flux through the ring of $\Phi / \Phi_{0}=$ $\pm \frac{\pi}{2}$ there still exists channels available for the conduction of electrons through the device, which implies the interference is only partially destructive. This is in contrast to the case of the normal ring [shown in Fig. 7(a)] where transmission is completely absent for this magnetic field strength.

We now consider the transmission probability for an $\mathrm{AB}$ ring in the topologically nontrivial regime with an applied magnetic field $(\Delta / t=0.1, \mu / t=1)$ shown in Fig. 7(c). This is characterized by the presence of a zero-energy state at $\Phi / \Phi_{0}=0$. The MZMs exhibit a linear energy dependence at magnetic flux about $\Phi / \Phi_{0}=n \pi$ where $n \in \mathbb{Z}$. Just as in 

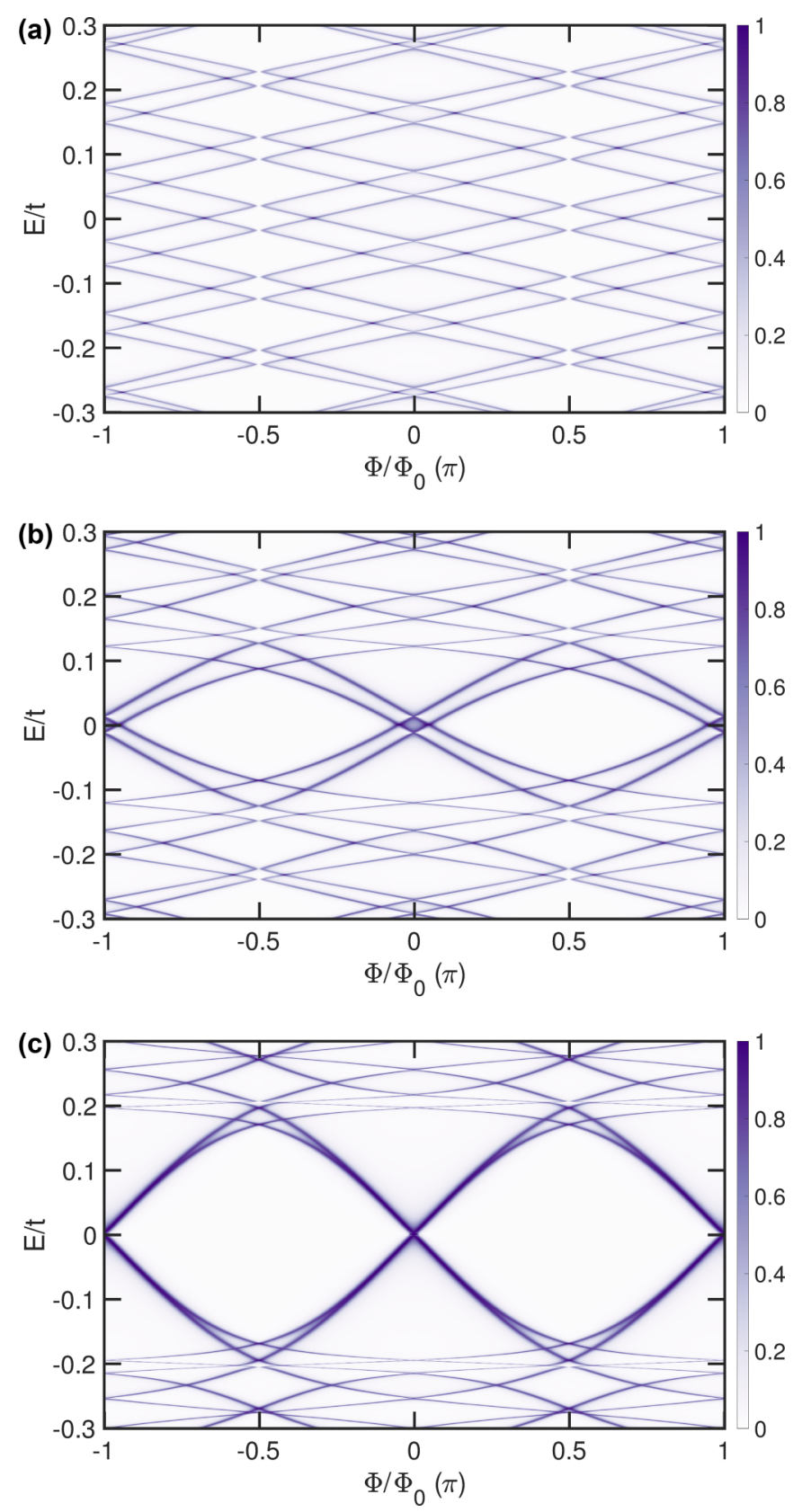

FIG. 7. Transmission probability of an $\mathrm{AB}$ ring as a function magnetic flux $\Phi$ and energy $E$ for various values of $p$-wave pairing amplitude (a) $\Delta / t=0$, (b) $\Delta / t=0.05$, and (c) $\Delta / t=0.1$. The AB ring has an on-site potential $\mu / t=1.0$ and normal leads are attached with a coupling strength of $\tau / t=0.3$.

Fig. 7(b), the ground state of the system is protected against destructive interference at $\Phi / \Phi_{0}=\frac{\pi}{2}$. However, now this behavior extends to the quasiparticle states at both higher and lower energies $(|E| / t \gtrsim 0.2)$. Although the splitting of the zero-energy state shows the degeneracy of the ground state is broken by an applied magnetic field, these states are still protected against destructive interference, which is something that is absent in the topologically trivial regime.

Figure 8 gives the Andreev and direct transmission for an $\mathrm{AB}$ ring when $\mu / t=1$. Crossed Andreev transmission is absent from this figure because it is found to be zero for
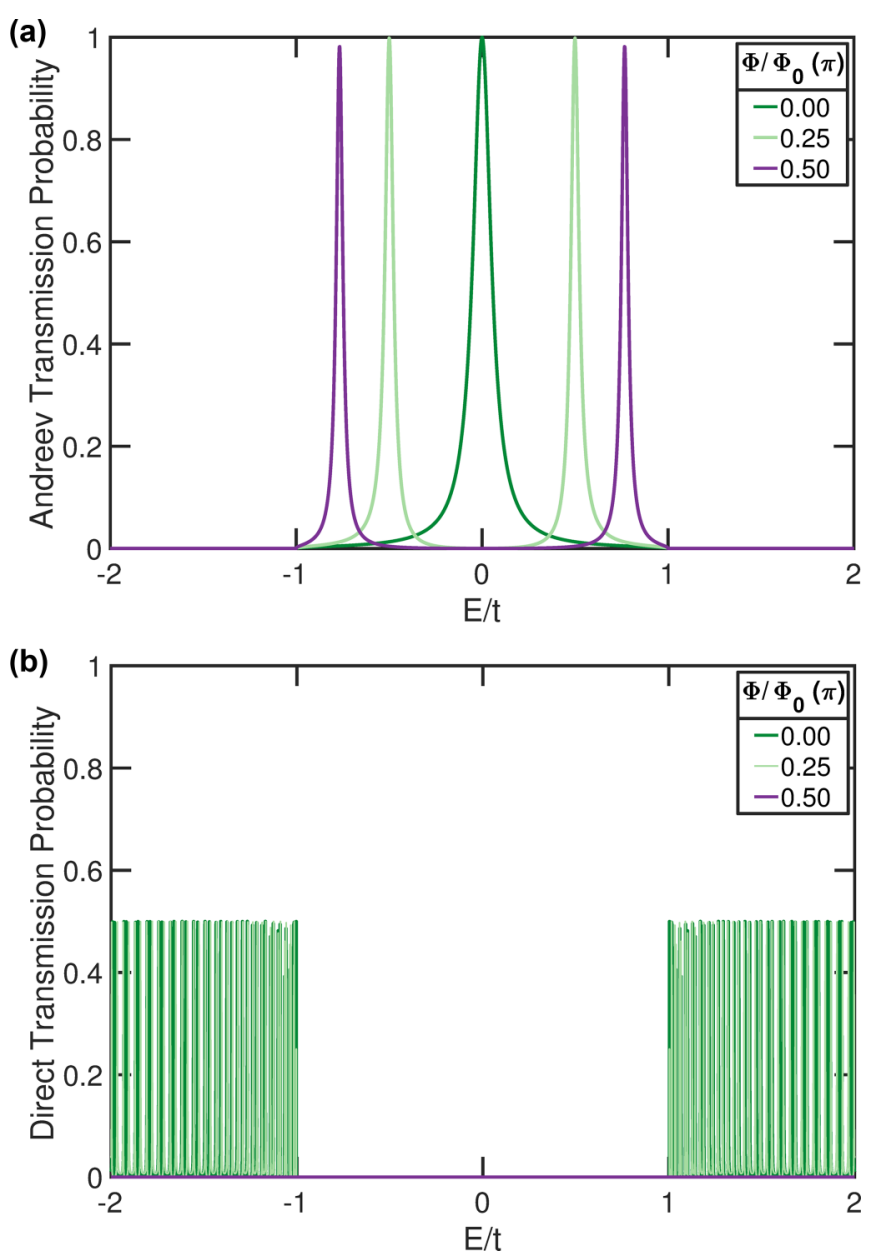

FIG. 8. Contributions to the transport probability from (a) Andreev and (b) direct transmission for an $\mathrm{AB}$ ring with $50 \times 2$ sites as a function of energy $E$. Here the on-site potential is $\mu / t=1$. The $\mathrm{AB}$ ring has a $p$-wave pairing amplitude $\Delta / t=1.0$ and normal leads are attached with a coupling strength of $\tau / t=0.3$.

these parameters. At a magnetic flux $\Phi / \Phi_{0}=0$ we see a zero-energy peak for Andreev transmission [Fig. 8(a)], as well as contributions to the direction transmission from quasiparticle states [Fig. 8(b)]. As the magnetic flux through the ring is varied such that the condition for completely destructive interference is met $\left(\Phi / \Phi_{0}=\frac{\pi}{2}\right)$ we see the suppression of all direct transmission, which is consistent with the behavior typically observed in $\mathrm{AB}$ interference. In contrast to this the Andreev transmission is found to persist at $\Phi / \Phi_{0}=\frac{\pi}{2}$. As with Fig. 7 we see a splitting in the zero-energy state that depends on the magnetic flux through the ring and increases with $\Phi / \Phi_{0}$ between 0 and $\frac{\pi}{2}$. This highlights that it is the Andreev transmission that is protected against destructive interference in the $\mathrm{AB}$ ring. The extent to which Andreev transmission is protected against destructive interference is shown in Fig. 9, where the transmission probability is plotted as a function of energy for several different values of the onsite potential and a magnetic flux $\Phi / \Phi_{0}=\frac{\pi}{2}$. As the value of the on-site potential is increased and the system moves toward the topologically trivial regime, the width of the subgap states becomes vanishingly small. 


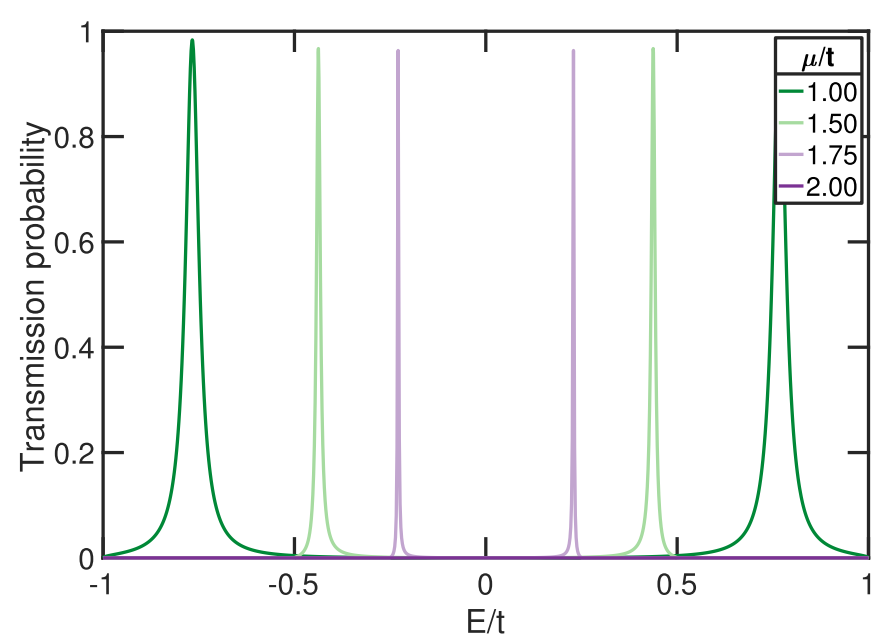

FIG. 9. Transmission probability of an $\mathrm{AB}$ ring with $50 \times 2$ sites as a function of energy $E$ for various on-site potentials $\mu$ and a magnetic flux of $\Phi / \Phi_{0}=\frac{\pi}{2}$. The $\mathrm{AB}$ ring has a $p$-wave pairing amplitude $\Delta / t=1.0$ and normal leads are attached with a coupling strength of $\tau / t=0.3$. When $\mu / t=2$ the transmission is zero for all energies.

The absence of destructive interference for an $\mathrm{AB}$ ring in the nontrivial regime, as shown in Figs. 7(c) and 9, is of particular interest. Previous studies have concluded that electrons move through MZMs preserving phase coherence [57]. This would suggest that transport should be suppressed at half integer multiples of magnetic flux quanta (e.g., $\Phi / \Phi_{0}= \pm \frac{\pi}{2}$ ), as is typically true for the AB effect. Instead we see a splitting in energy of the topologically nontrivial state and a shift in the energy of this state away from zero, in response to changing the magnetic flux threading the loop. This raises questions as to whether the topological protection of the MZMs is still preserved in such a situation. It also suggests that the response of such a circuit to a magnetic field can be used as a probe of topologically trivial Andreev bound states.

\section{EFFECTS OF DISORDER}

Experimentally realisable systems will contain noise which may perturb the system. One way this can occur is through disorder within the system itself. To study the effect of disorder on our $\mathrm{AB}$ rings, we introduce to each site $n$ a quasirandom on-site energy $w_{n}$ which is obtained from a uniform distribution [58] centered about zero such that $w_{n} \in(-1,1)$. The magnitude of the disorder is controlled via a disorder amplitude $P$ such that the on-site term for Eq. (1) becomes $\mu_{n}=\mu+\left(P w_{n}\right)$.

Electron transport in nanoscale devices is often limited by random offset charges stemming from surfaces and interfaces within the device [59-64]. This background charge disorder can either be static (approximately constant once the device is cooled to base temperature) or dynamic during the measurement period, resulting in an ensemble averaged signal over multiple disorder realizations.

For an $\mathrm{AB}$ ring without an applied magnetic field transmission is unchanged by the inclusion of weak disorder. The MZM in such a system is robust against both weak dynamic

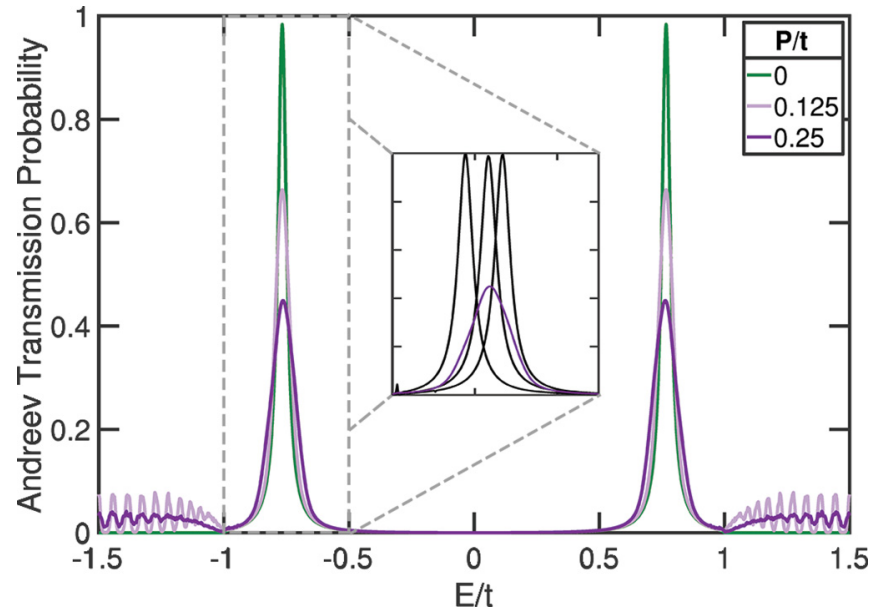

FIG. 10. Transmission in an $\mathrm{AB}$ ring with $\Delta / t=\mu / t=1.0,50$ sites and a magnetic field of $\phi / \phi_{0}=\frac{\pi}{2}$, for several values of disorder strength $P$. Each disorder value in this figure has been averaged over 1000 configurations. The inset shows the a sample of the static disorder realisations together with the dynamic disorder result for a disorder strength of $P / t=0.25$.

and static disorder, as seen in literature for 1D wires containing MZMs [65]. However the same is not true for an AB ring in the presence of a magnetic field. Figure 10 shows the effect of introducing a dynamic disorder scheme into the $\mathrm{AB}$ ring with magnetic field applied. The disorder has been introduced in the topologically nontrivial regime where $\phi / \phi_{0}=\frac{\pi}{2}$ in the form of a random on-site variation. We see that increasing the strength of the disorder broadens the transmission peak and reduces the peak height of the system with dynamic disorder. This reduction in averaged peak height is caused by a disorder induced shifting of the energy values required to achieve peak transmission. This effect is illustrated in the inset, which shows the average transmission for a disorder strength of $P / t=0.25$, with several of the static disorder realizations which have been averaged to get the dynamic disorder result. Note that the peak height for static disorder is unchanged by the strength of the disorder.

The movement of the peaks in the inset of Fig. 10 under disorder may be due to the fluctuations in the on-site potential, which causes regions of the ring to transition either deeper into or out of the topologically nontrivial regime. For a system being pushed deeper into the topologically nontrivial regime, the superconducting gap would become larger as in Fig. 4. For a system with magnetic field applied, we see in Fig. 9 that decreasing the on-site potential causes the peaks to move to higher energies. However, that there still exist conductance channels with an applied magnetic field of $\phi / \phi_{0}=\frac{\pi}{2}$ and in the presence of disorder suggests that these channels are robust against weak disorder.

As the type of disorder affects the resultant transmission peaks, one would expect experimental signatures of the type seen in Fig. 7(c) to change depending on the type of disorder introduced. Static disorder would cause the separation between the transmission peaks to fluctuate, with the fluctuations increasing in magnitude with disorder strength. However for a system with dynamic disorder, one would expect the 
separation between the transmission peaks to remain relatively constant, but rather the peak amplitude would be decreased. In both cases, for weak disorder the conduction channels persist in the presence of partially destructive $A B$ interference.

\section{CONCLUSION}

We used the NEGF formalism to study the transport properties of an $\mathrm{AB}$ ring comprised of two Kitaev chains coupled at either end by a normal link. We observed the effect of electron (hole) interference on the zero-bias transmission of this $\mathrm{AB}$ ring, which is present when the magnetic flux through the ring is nonzero. We have shown how the MZMs in this $\mathrm{AB}$ ring change with on-site potential $\mu$, the $p$-wave pairing amplitude $\Delta$, and the magnetic flux through the ring $\Phi$. Each of these parameters has been shown to have a unique effect on the MZMs. Having control of the physical parameters $\mu$, $\Delta$, and $\Phi$ in an experiment therefore allows the MZMs to be probed in a controllable way.

Furthermore, we have observed the transmission probability of electrons and holes through an $\mathrm{AB}$ ring with MZMs to be persistent even when the condition for completely destructive interference are met. The power of the NEGF formalism as a tool for investigating this phenomenon is highlighted where the transmission probabilities are divided into three separate contributions: Andreev and crossed Andreev reflection and direct transmission. We have thereby demonstrated that transmission through MZMs in an $\mathrm{AB}$ ring is mediated by Andreev reflection at the normal/superconductor interface. The dependence of the $\mathrm{AB}$ interference on the parameters of the nanowires is therefore a useful probe of Majorana fermions in condensed matter systems.

The mechanism which provides the above gap states protection from the $\mathrm{AB}$ remains unclear. We believe this issue requires further investigation using different tools than those used here and therefore we leave it for further work.

\section{ACKNOWLEDGMENTS}

The authors acknowledge useful discussions with $\mathrm{S}$. Rachel. The authors would also like to thank J. A. Vaitkus and M. J. Cyster for providing MATLAB code. This work was supported by the Australian Research Council through Grants No. CE170100039 (FLEET) and No. CE170100009 (EQUS). C.M. is supported by the Swiss National Science Foundation through NCCR Quantum Science and Technology (QSIT). B.M. would like to acknowledge funding from the Ministry of Human Resource Development (MHRD), Government of India, Grant No. STARS/APR2019/NS/226/FS under the STARS scheme and the Visvesvaraya Scheme of the Ministry of Electronics and Information Technology (MeiTy), Government of India. This research was undertaken with the assistance of resources from the National Computational Infrastructure, which is supported by the Australian Government.

\section{APPENDIX A: PARTICLE-HOLE SYMMETRY}

The Kitaev Hamiltonian when expressed in the BdG form exhibits particle-hole symmetry (PHS). PHS represents a symmetry in the behavior of electrons and holes and leads to a symmetry in the eigenspectrum around zero energy. As we are investigating a superconducting system, the Hamiltonian contains a coupling term between particles and holes, which is best described in the BdG formalism. Employing the Nambu basis, we group creation and annihilation operators together as

$$
\begin{aligned}
& C^{\dagger}=\left\{c_{1}^{\dagger}, \ldots, c_{n}^{\dagger}, c_{1}, \ldots, c_{n}\right\}, \\
& C=\left\{c_{1}, \ldots, c_{n}, c_{1}^{\dagger}, \ldots, c_{n}^{\dagger}\right\}^{T} .
\end{aligned}
$$

This notation then allows us to write the BdG Hamiltonian as

$$
\hat{H}=\frac{1}{2} C^{\dagger} H_{\mathrm{BdG}} C
$$

with the BdG Hamiltonian coefficient matrix for the Kitaev chain:

$$
H_{\mathrm{BdG}}=\left[\begin{array}{cc}
H & \Delta \\
-\Delta^{*} & -H^{*}
\end{array}\right] .
$$

Here PHS manifests directly in the symmetry between the particle and hole sectors of the Hamiltonian.

\section{APPENDIX B: DERIVATION OF THE COMPONENTS OF CURRENT IN A SYSTEM WITH PARTICLE-HOLE SYMMETRY}

The conventional equation for calculating the transmission of electrons through a device is given in Eq. (4). When applied to a system in conjunction with the BdG form of the Hamiltonian we must also take into consideration the transmission probability for holes. This allows for the study of more transport modes than just the conventional transport of electrons through a device [66-68]. Andreev and crossed Andreev transmission allow for the reflection of an electron from an interface as a hole both locally and nonlocally respectively $[13,69,70]$. In order to probe the role these processes play in this work, Eqs. (8)-(10) were applied. Although here we discuss how Eqs. (8)-(10) can be derived from the Landauer current equations, it is important to note that they are themselves particle transmission functions and not current equations. The distinction is that the transmission functions are independent of the Fermi distribution function (and therefore temperature and lead Fermi levels). These equations are derived from the Landauer current equation:

$$
I_{\mathrm{L}}=\frac{q}{h} \operatorname{Tr}\left[-i \Sigma_{\mathrm{L}}^{<} A-\Gamma_{\mathrm{L}} G^{n}\right],
$$

where $\Gamma_{\alpha}=\Gamma_{\alpha}(E)$ is the broadening matrix for lead $\alpha$. We also have

$$
G^{n}=-i G^{<}=-i G^{R}\left(\Sigma_{\mathrm{L}}^{<}+\Sigma_{\mathrm{R}}^{<}\right) G^{A} .
$$

and the spectral function is defined as

$$
A=G^{R} \Gamma G^{A} .
$$

Applying these definitions to the Landauer current equation, we arrive at

$$
I_{\mathrm{L}}=\frac{q}{h} \operatorname{Tr}\left[-i \Sigma_{\mathrm{L}}^{<} G^{R}\left(\Gamma_{\mathrm{L}}+\Gamma_{\mathrm{R}}\right) G^{A}+i \Gamma_{\mathrm{L}} G^{R}\left(\Sigma_{\mathrm{L}}^{<}+\Sigma_{\mathrm{R}}^{<}\right) G^{A}\right] .
$$

As we are working in the BdG formalism which utilizes the Nambu (particle-hole) basis, we must take both particle and 
hole transmission into account. With the application of normal leads there is no coupling between particles and holes and as such the in-scattering matrix $\left(\Sigma^{<}\right)$becomes block diagonal (one block for electron scattering and one for hole scattering). We are therefore able to write the in-scattering matrix for lead $\alpha$ as

$$
\Sigma_{\alpha}^{<}=i \Gamma_{\alpha}^{\mathrm{e}} f_{\alpha}^{\mathrm{e}}+i \Gamma_{\alpha}^{\mathrm{h}} f_{\alpha}^{\mathrm{h}},
$$

where the superscript e (h) denotes the electron (hole) component in the Nambu basis and $f_{\alpha}(E)=\left(e^{\left(E+E_{F}\right) / k T}+1\right)^{-1}$ is the Fermi distribution for the $\alpha$ lead with Fermi energy $E_{F}$ at temperature $T$. Combining Eqs. (B4) and (B5) we arrive at

$$
I_{\mathrm{L}}^{\mathrm{e}(\mathrm{h})}=\frac{q}{h} \operatorname{Tr}\left[\left(\Gamma_{\mathrm{L}}^{\mathrm{e}(\mathrm{h})} f_{\mathrm{L}}^{\mathrm{e}(\mathrm{h})}+\Gamma_{\mathrm{L}}^{\mathrm{h}(\mathrm{e})} f_{\mathrm{L}}^{\mathrm{h}(\mathrm{e})}\right) G^{R}\left(\Gamma_{\mathrm{L}}+\Gamma_{\mathrm{R}}\right) G^{A}\right.
$$

$$
\begin{aligned}
& -\Gamma_{\mathrm{L}} G^{R}\left\{\left(\Gamma_{\mathrm{L}}^{\mathrm{e}(\mathrm{h})} f_{\mathrm{L}}^{\mathrm{e}(\mathrm{h})}+\Gamma_{\mathrm{L}}^{\mathrm{h}(\mathrm{e})} f_{\mathrm{L}}^{\mathrm{h}(\mathrm{e})}\right)\right. \\
& \left.\left.+\left(\Gamma_{\mathrm{R}}^{\mathrm{e}(\mathrm{h})} f_{\mathrm{R}}^{\mathrm{e}(\mathrm{h})}+\Gamma_{\mathrm{R}}^{\mathrm{h}(\mathrm{e})} f_{\mathrm{R}}^{\mathrm{h}(\mathrm{e})}\right)\right\} G^{A}\right] .
\end{aligned}
$$

which after some algebraic manipulation returns

$$
\begin{aligned}
I_{\mathrm{L}}^{\mathrm{e}(\mathrm{h})}= & \frac{q}{h} \operatorname{Tr}\left[\Gamma_{\mathrm{L}}^{\mathrm{e}(\mathrm{h})} G^{R} \Gamma_{R}^{\mathrm{e}(\mathrm{h})} G^{A}\left(f_{\mathrm{L}}^{\mathrm{e}(\mathrm{h})}-f_{\mathrm{R}}^{\mathrm{e}(\mathrm{h})}\right)\right. \\
& +\Gamma_{\mathrm{L}}^{\mathrm{e}(\mathrm{h})} G^{R} \Gamma_{\mathrm{L}}^{\mathrm{h}(\mathrm{e})} G^{A}\left(f_{\mathrm{L}}^{\mathrm{e}(\mathrm{h})}-f_{\mathrm{L}}^{\mathrm{h}(\mathrm{e})}\right) \\
& \left.+\Gamma_{\mathrm{L}}^{\mathrm{e}(\mathrm{h})} G^{R} \Gamma_{\mathrm{R}}^{\mathrm{h}(\mathrm{e})} G^{A}\left(f_{\mathrm{L}}^{\mathrm{e}(\mathrm{h})}-f_{\mathrm{R}}^{\mathrm{h}(\mathrm{e})}\right)\right] .
\end{aligned}
$$

As we only want to study the transmission, rather than the current, we can neglect the constant factor as well as the Fermi windowing function, after which we arrive at the transmission equations given in Eqs. (8)-(10).
[1] E. Majorana and L. Maiani, A symmetric theory of electrons and positrons, in Ettore Majorana Scientific Papers (Springer, Berlin, 2006), pp. 201-233.

[2] A. Y. Kitaev, Unpaired Majorana fermions in quantum wires, Phys. Usp. 44, 131 (2001).

[3] J. Alicea, Y. Oreg, G. Refael, F. Von Oppen, and M. P. A. Fisher, Non-Abelian statistics and topological quantum information processing in 1D wire networks, Nat. Phys. 7, 412 (2011).

[4] B. Lian, X. Q. Sun, A. Vaezi, X. L. Qib, and S. C. Zhang, Topological quantum computation based on chiral Majorana fermions, Proc. Natl. Acad. Sci. USA 115, 10938 (2018).

[5] D. A. Ivanov, Non-Abelian Statistics of Half-Quantum Vortices in $p$-Wave Superconductors, Phys. Rev. Lett. 86, 268 (2001).

[6] G. E. Volovik, Fermion zero modes on vortices in chiral superconductors, JETP Lett. 70, 609 (1999).

[7] N. Read and D. Green, Paired states of fermions in two dimensions with breaking of parity and time-reversal symmetries and the fractional quantum Hall effect, Phys. Rev. B 61, 10267 (2000).

[8] B. van Heck, A. R. Akhmerov, F. Hassler, M. Burrello, and C. W. J. Beenakker, Coulomb-assisted braiding of Majorana fermions in a Josephson junction array, New J. Phys. 14, 035019 (2012).

[9] L. H. Kauffman, in Logic and Algebraic Structures in Quantum Computing, edited by J. Chubb, A. Eskandarian, and V. Harizanov (Cambridge University Press, Cambridge, 2016), pp. 223-336.

[10] A. Yu. Kitaev, Fault-tolerant quantum computation by anyons, Ann. Phys. 303, 2 (2003).

[11] V. Mourik, K. Zuo, S. M. Frolov, S. R. Plissard, E. P. A. M. Bakkers, and L. P. Kouwenhoven, Signatures of Majorana fermions in hybrid superconductor-semiconductor nanowire devices, Science 336, 1003 (2012).

[12] H. Zhang, C.-X. Liu, S. Gazibegovic, D. Xu, J. A. Logan, G. Wang, N. van Loo, J. D. S. Bommer, M. W. A. de Moor, D. Car, R. L. M. Op het Veld, P. J. van Veldhoven, S. Koelling, M. A. Verheijen, M. Pendharkar, D. J. Pennachio, B. Shojaei, J. S. Lee, C. J. Palmstrøm, E. P. A. M. Bakkers, S. D. Sarma, and L. P. Kouwenhoven, Quantized Majorana conductance, Nature (London) 556, 74 (2018).

[13] K. T. Law, P. A. Lee, and T. K. Ng, Majorana Fermion Induced Resonant Andreev Reflection, Phys. Rev. Lett. 103, 237001 (2009).
[14] A. Das, Y. Ronen, Y. Most, Y. Oreg, M. Heiblum, and H. Shtrikman, Zero-bias peaks and splitting in an Al-InAs nanowire topological superconductor as a signature of Majorana fermions, Nat. Phys. 8, 887 (2012).

[15] M. T. Deng, C. L. Yu, G. Y. Huang, M. Larsson, P. Caroff, and H. Q. Xu, Anomalous zero-bias conductance peak in a Nb-InSb nanowire- Nb hybrid device, Nano Lett. 12, 6414 (2012).

[16] L. P. Rokhinson, X. Liu, and J. K. Furdyna, The fractional a.c. Josephson effect in a semiconductor-superconductor nanowire as a signature of Majorana particles, Nat. Phys. 8, 795 (2012).

[17] M. T. Deng, S. Vaitiekenas, E. B. Hansen, J. Danon, M. Leijnse, K. Flensberg, J. Nygård, P. Krogstrup, and C. M. Marcus, Majorana bound state in a coupled quantum-dot hybrid-nanowire system, Science 354, 1557 (2016).

[18] C.-X. Liu, J. D. Sau, and S. Das Sarma, Distinguishing topological Majorana bound states from trivial Andreev bound states: Proposed tests through differential tunneling conductance spectroscopy, Phys. Rev. B 97, 214502 (2018).

[19] C.-X. Liu, J. D. Sau, T. D. Stanescu, and S. Das Sarma, Andreev bound states versus Majorana bound states in quantum dot-nanowire-superconductor hybrid structures: Trivial versus topological zero-bias conductance peaks, Phys. Rev. B 96, 075161 (2017).

[20] C. Moore, T. D. Stanescu, and S. Tewari, Two-terminal charge tunneling: Disentangling Majorana zero modes from partially separated Andreev bound states in semiconductorsuperconductor heterostructures, Phys. Rev. B 97, 165302 (2018).

[21] M.-T. Deng, S. Vaitiekenas, E. Prada, P. San-Jose, J. Nygård, P. Krogstrup, R. Aguado, and C. M. Marcus, Nonlocality of Majorana modes in hybrid nanowires, Phys. Rev. B 98, 085125 (2018).

[22] C.-K. Chiu and S. Das Sarma, Fractional Josephson effect with and without Majorana zero modes, Phys. Rev. B 99, 035312 (2019).

[23] C. Moore, C. Zeng, T. D. Stanescu, and S. Tewari, Quantized zero-bias conductance plateau in semiconductorsuperconductor heterostructures without topological Majorana zero modes, Phys. Rev. B 98, 155314 (2018).

[24] L. Chen, W. LiMing, J.-H. Huang, C. Yin, and Z.-Y. Xue, Majorana zero modes on a one-dimensional chain for quantum computation, Phys. Rev. A 90, 012323 (2014). 
[25] J. Alicea, New directions in the pursuit of Majorana fermions in solid state systems, Rep. Prog. Phys. 75, 076501 (2012).

[26] M. Lee, H. Khim, and M.-S. Choi, Anomalous crossed Andreev reflection in a mesoscopic superconducting ring hosting Majorana fermions, Phys. Rev. B 89, 035309 (2014).

[27] R. J. Doornenbal, G. Skantzaris, and H. T. C. Stoof, Conductance of a finite Kitaev chain, Phys. Rev. B 91, 045419 (2015).

[28] L. Weithofer, P. Recher, and T. L. Schmidt, Electron transport in multiterminal networks of Majorana bound states, Phys. Rev. B 90, 205416 (2014).

[29] J. Danon, A. B. Hellenes, E. B. Hansen, L. Casparis, A. P. Higginbotham, and K. Flensberg, Nonlocal Conductance Spectroscopy of Andreev Bound States: Symmetry Relations and BCS Charges, Phys. Rev. Lett. 124, 036801 (2020).

[30] B. van Heck, F. Hassler, A. R. Akhmerov, and C. W. J. Beenakker, Coulomb stability of the $4 \pi$-periodic Josephson effect of Majorana fermions, Phys. Rev. B 84, 180502(R) (2011).

[31] Q.-B. Zeng, S. Chen, L. You, and R. Lü, Transport through a quantum dot coupled to two Majorana bound states, Front. Phys. 12, 127302 (2017).

[32] A. R. Akhmerov, J. P. Dahlhaus, F. Hassler, M. Wimmer, and C. W. J. Beenakker, Quantized Conductance at the Majorana Phase Transition in a Disordered Superconducting Wire, Phys. Rev. Lett. 106, 057001 (2011).

[33] A. M. Whiticar, A. Fornieri, E. C. T. O'Farrell, A. C. C. Drachmann, T. Wang, C. Thomas, S. Gronin, R. Kallaher, G. C. Gardner, M. J. Manfra, C. M. Marcus, and F. Nichele, Coherent transport through a Majorana island in an Aharonov-Bohm interferometer, Nat. Commun. 11, 3212 (2020).

[34] K. M. Tripathi, S. Das, and S. Rao, Fingerprints of Majorana Bound States in Aharonov-Bohm Geometry, Phys. Rev. Lett. 116, 166401 (2016).

[35] E.-M. Shang, Y.-M. Pan, L.-B. Shao, and B.-G. Wang, Detection of Majorana fermions in an Aharonov-Bohm interferometer, Chin. Phys. B 23, 057201 (2014).

[36] C. Benjamin and J. K. Pachos, Detecting Majorana bound states, Phys. Rev. B 81, 085101 (2010).

[37] E. B. Hansen, J. Danon, and K. Flensberg, Phase-tunable Majorana bound states in a topological N-SNS junction, Phys. Rev. B 93, 094501 (2016).

[38] C. Jiang and Y.-S. Zheng, Fano effect in the Andreev reflection of the Aharonov-Bohm-Fano ring with Majorana bound states, Solid State Commun. 212, 14 (2015).

[39] T. Haug, R. Dumke, L.-C. Kwek, and L. Amico, Andreevreflection and Aharonov-Bohm dynamics in atomtronic circuits, Quantum Sci. Technol. 4, 045001 (2019).

[40] T. Haug, H. Heimonen, R. Dumke, L.-C. Kwek, and L. Amico, Aharonov-Bohm effect in mesoscopic Bose-Einstein condensates, Phys. Rev. A 100, 041601(R) (2019).

[41] T. Haug, R. Dumke, L.-C. Kwek, and L. Amico, Topological pumping in Aharonov-Bohm rings, Commun. Phys. 2, 127 (2019).

[42] L. Qin, X.-Q. Li, A. Shnirman, and G. Schön, Transport signatures of a Majorana qubit and read-out-induced dephasing, New J. Phys. 21, 043027 (2019).

[43] V. V. Val'kov, M. Y. Kagan, and S. V. Aksenov, Fano effect in Aharonov-Bohm ring with topologically superconducting bridge, J. Phys.: Condens. Matter 31, 225301 (2019).
[44] S. V. Aksenov and M. Y. Kagan, Collapse of the Fano resonance caused by the nonlocality of the Majorana state, JETP Lett. 111, 286 (2020).

[45] S. Park, J. E. Moore, and H.-S. Sim, Absence of the AharonovBohm effect of chiral Majorana fermion edge states, Phys. Rev. B 89, 161408(R) (2014).

[46] L. Fu and C. L. Kane, Probing Neutral Majorana Fermion Edge Modes with Charge Transport, Phys. Rev. Lett. 102, 216403 (2009).

[47] A. A. Zvyagin, Majorana bound states in the finite-length chain, Low Temp. Phys. 41, 625 (2015).

[48] N. Leumer, M. Marganska, B. Muralidharan, and M. Grifoni, Exact eigenvectors and eigenvalues of the finite Kitaev chain and its topological properties, J. Phys.: Condens. Matter 32, 445502 (2020).

[49] Y. J. Zhang, S. Zhang, G. Y. Yi, C. Jiang, and W. J. Gong, Interference effects in the Aharonov-Bohm geometries with Majorana bound states, Superlattices Microstruct. 113, 25 (2018).

[50] S. Datta, Quantum Transport: Atom to Transistor (Cambridge University Press, Cambridge, 2005).

[51] M. Lundstrom, Fundamentals of Carrier Transport (Cambridge University Press, Cambridge, 2000).

[52] R. Peierls, Zur Theorie des Diamagnetismus von Leitungselektronen, Z. Phys. 80, 763 (1933).

[53] M. M. Maśka and M. Mierzejewski, Upper critical field for anisotropic superconductivity: A tight-binding approach, Phys. Rev. B 64, 064501 (2001).

[54] D. R. Hofstadter, Energy levels and wave functions of Bloch electrons in rational and irrational magnetic fields, Phys. Rev. B 14, 2239 (1976).

[55] P. Bosland, F. Guemas, M. Juillard, M. Couach, and A. F. Khoder, NbTiN superconducting thin films prepared by magnetron sputtering, in Third European Particle Accelerator Conference, Berlin, 24-28 March 1992, EPAC 92 (Editions Frontieres, Lausanne, 1992), pp. 1316-1318.

[56] R. Di Leo, A. Nigro, G. Nobile, and R. Vaglio, Niboiumtitanium nitride thin films for superconducting rf accelerator cavities, J. Low Temp. Phys. 78, 41 (1990).

[57] L. Fu, Electron Teleportation via Majorana Bound States in a Mesoscopic Superconductor, Phys. Rev. Lett. 104, 056402 (2010).

[58] N. M. Gergs, L. Fritz, and D. Schuricht, Topological order in the Kitaev/Majorana chain in the presence of disorder and interactions, Phys. Rev. B 93, 075129 (2016).

[59] A. Pourkabirian, M. V. Gustafsson, G. Johansson, J. Clarke, and P. Delsing, Nonequilibrium Probing of Two-Level Charge Fluctuators Using the Step Response of a Single-Electron Transistor, Phys. Rev. Lett. 113, 256801 (2014).

[60] A. B. Zorin, F.-J. Ahlers, J. Niemeyer, T. Weimann, H. Wolf, V. A. Krupenin, and S. V. Lotkhov, Background charge noise in metallic single-electron tunneling devices, Phys. Rev. B 53, 13682 (1996).

[61] N. M. Zimmerman, W. H. Huber, B. Simonds, E. Hourdakis, A. Fujiwara, Y. Ono, Y. Takahashi, H. Inokawa, M. Furlan, and M. W. Keller, Why the long-term charge offset drift in $\mathrm{Si}$ single-electron tunneling transistors is much smaller (better) than in metal-based ones: Two-level fluctuator stability, J. Appl. Phys. 104, 033710 (2008). 
[62] K. Cedergren, R. Ackroyd, S. Kafanov, N. Vogt, A. Shnirman, and T. Duty, Insulating Josephson Junction Chains as Pinned Luttinger Liquids, Phys. Rev. Lett. 119, 167701 (2017).

[63] C. Müller, J. H. Cole, and J. Lisenfeld, Towards understanding two-level-systems in amorphous solids: Insights from quantum circuits, Rep. Prog. Phys. 82, 124501 (2019).

[64] M. V. Gustafsson, A. Pourkabirian, G. Johansson, J. Clarke, and P. Delsing, Thermal properties of charge noise sources, Phys. Rev. B 88, 245410 (2013).

[65] S. Lieu, D. K. K. Lee, and J. Knolle, Disorder protected and induced local zero-modes in longer-range Kitaev chains, Phys. Rev. B 98, 134507 (2018).

[66] P. Sriram, S. S. Kalantre, K. Gharavi, J. Baugh, and B. Muralidharan, Supercurrent interference in semiconductor nanowire Josephson junctions, Phys. Rev. B 100, 155431 (2019).

[67] Y. Wei, J. Wang, H. Guo, H. Mehrez, and C. Roland, Resonant Andreev reflections in superconductor-carbon-nanotube devices, Phys. Rev. B 63, 195412 (2001).

[68] Q.-f. Sun, J. Wang, and T.-h. Lin, Resonant Andreev reflection in a normal-metal-quantum-dot-superconductor system, Phys. Rev. B 59, 3831 (1999).

[69] S.-X. Wang, Y.-X. Li, and J.-J. Liu, Resonant Andreev reflection in a normal-metal/quantum-dot/superconductor system with coupled Majorana bound states, Chin. Phys. B 25, 037304 (2016).

[70] J. Nilsson, A. R. Akhmerov, and C. W. J. Beenakker, Splitting of a Cooper Pair by a Pair of Majorana Bound States, Phys. Rev. Lett. 101, 120403 (2008). 